\title{
SINGULAR INTEGRALS ON SIERPINSKI GASKETS
}

\author{
VAsilis Chousionis
}

Abstract

We construct a class of singular integral operators associated with homogeneous Calderón-Zygmund standard kernels on $d$-dimensional, $d<1$, Sierpinski gaskets $E_{d}$. These operators are bounded in $L^{2}\left(\mu_{d}\right)$ and their principal values diverge $\mu_{d}$ almost everywhere, where $\mu_{d}$ is the natural ( $d$-dimensional) measure on $E_{d}$.

\section{Introduction}

Given a Radon measure $\mu$ on $\mathbb{R}^{n}$ and a continuously differentiable kernel $K: \mathbb{R}^{n} \times \mathbb{R}^{n} \backslash\{(x, y): x=y\} \rightarrow \mathbb{R}$ that satisfies the antisymmetry condition

$$
K(x, y)=-K(y, x) \text { for } x, y \in \mathbb{R}^{n}, x \neq y,
$$

the singular integral operator $T$ associated with $K$ and $\mu$ is formally given by

$$
T(f)(x)=\int K(x, y) f(y) d \mu y .
$$

Notice that the above integral does not usually exist when $x \in \operatorname{spt} \mu$. The truncated singular integral operators $T_{\varepsilon}, \varepsilon>0$;

$$
T_{\varepsilon}(f)(x)=\int_{|x-y|>\varepsilon} K(x, y) f(y) d \mu y,
$$

are considered in order to overcome this obstacle. In the same vein one considers the maximal operator $T^{*}$

$$
T^{*}(f)(x)=\sup _{\varepsilon>0}\left|T_{\varepsilon}(f)(x)\right|
$$

and the principal values of $T(f)$ at every $x \in \mathbb{R}^{n}$ which, if they exist, are given by

$$
\text { p.v. } T(f)(x)=\lim _{\varepsilon \rightarrow 0} T_{\varepsilon}(f)(x) .
$$

2000 Mathematics Subject Classification. Primary: 42B20.

Key words. Singular integrals, self similar sets.

The author is supported by the Finnish Graduate School in Mathematical Analysis. 
The singular integral operator $T$ associated with $\mu$ and $K$ is said to be bounded in $L^{2}(\mu)$ if there exists some constant $C>0$ such that for $f \in L^{2}(\mu)$ and $\varepsilon>0$

$$
\int\left|T_{\varepsilon}(f)\right|^{2} d \mu \leq C \int|f|^{2} d \mu .
$$

The natural question as to whether the $L^{2}(\mu)$-boundedness of the operator $T$ forces its principal values to exist $\mu$ almost everywhere has been considered in many papers (see e.g. $[\mathbf{M M}],[\mathbf{M M V}],[\mathbf{T}],[\mathbf{D 2}],[\mathbf{H u}]$, $[\mathbf{M V}]$ and $[\mathbf{P r}])$. Even when $\mu$ is an $m$-dimensional Ahlfors-David (AD) regular measure in $\mathbb{R}^{n}$ :

$$
C^{-1} r^{m} \leq \mu(B(x, r)) \leq C r^{m} \text { for } x \in \operatorname{spt} \mu, 0<r<\operatorname{diam}(\operatorname{spt} \mu),
$$

and $K$ is any of the coordinate Riesz kernels:

$$
R_{i}^{m}(x, y)=\frac{x_{i}-y_{i}}{|x-y|^{m+1}} \text { for } i=1 \ldots, n
$$

the question remains open for $m>1$.

When $m=1$, or equivalently in the case of the Cauchy transform, the above question has a positive answer by the results of Mattila, Melnikov and Verdera (see $[\mathbf{M M}]$ and $[\mathbf{M M V}]$ ). Later on, in $[\mathbf{T}]$, Tolsa improved the afore mentioned results by removing the Ahlfors-David regularity assumption.

In different settings the answer to the above question can be negative. Let $C$ be the 1-dimensional four corners Cantor set and $\mu$ its natural (1-dimensional Hausdorff) measure. David in [D2], constructed Calderón-Zygmund standard, or simply CZ standard, kernels that define operators bounded in $L^{2}(\mu)$ whose principal values fail to exist $\mu$ almost everywhere. Although David's kernels can be chosen odd or even are not homogeneous of degree -1 .

In this note we consider classical plane Sierpinski gaskets of Hausdorff dimension $d, 0<d<1$. For each of these $d$-AD regular sets $E_{d}$, we find families of $\mathrm{CZ}$ standard, smooth, and antisymmetric kernels of the form

$$
K(x, y)=\frac{\Omega((x-y) /|x-y|)}{h(|x-y|)}
$$

where $h$ is some increasing $C^{\infty}$ function satisfying the homogeneity condition

$$
h(r) \simeq r^{d}
$$

for $0<r<\operatorname{diam}\left(E_{d}\right)$ and $\Omega$ is odd on the unit circle $S^{1}$. If $\mu_{d}$ is the restriction of the $d$-dimensional Hausdorff measure on $E_{d}$, these kernels define singular integral operators bounded in $L^{2}\left(\mu_{d}\right)$ whose principal values 
diverge $\mu_{d}$ almost everywhere. The proof is based on the $T(1)$-theorem of David and Journé, proved in [DJ], and the symmetry properties of Sierpinski gaskets allowing heavy cancelations.

Remark 1.1. Unfortunately if in the above kernels we replace the function $h(r)$ by $r^{d}$, where in this case the kernels would be $d$-homogeneous in the classical sense, we cannot say if the corresponding operators are bounded (or not) in $L^{2}$. However their principal values diverge $\mu_{d}$ almost everywhere, as the proof of Section 4 goes through with no changes.

Remark 1.2. Modified slightly, the proof can be applied to many other symmetric self similar sets, e.g. the four corners Cantor sets with Hausdorff dimension less than 1 or the self similar sets discussed in [D2]. The dimensional restriction is essential for the proof and it is not known to us if there exist CZ standard kernels, of the same form as in (1.1), satisfying the homogeneity condition $h(r) \approx r$, that define singular integral operators bounded in $L^{2}$ but whose principal values diverge almost everywhere.

\section{Notation and setting}

Let $\lambda \in(0,1 / 3)$ and consider the following three similitudes (depending on $\lambda) s_{1}^{\lambda}, s_{2}^{\lambda}, s_{3}^{\lambda}: \mathbb{R}^{2} \rightarrow \mathbb{R}^{2}$

- $s_{1}^{\lambda}(x, y)=\lambda(x, y)$

- $s_{2}^{\lambda}(x, y)=\lambda(x, y)+(1-\lambda, 0)$

- $s_{3}^{\lambda}(x, y)=\lambda(x, y)+\left(\frac{1-\lambda}{2}, \frac{\sqrt{3}}{2}(1-\lambda)\right)$.

Let $I=\{1,2,3\}$ and $I^{*}=\bigcup_{n \geq 1} I^{n}$. The set $I^{*}$ can be partially ordered in the following way, for $\alpha, \beta \in I^{*}$,

$$
\alpha \prec \beta \Leftrightarrow \alpha \in I^{n}, \beta \in I^{k}, k \geq n \text { and } \beta\lfloor n=\alpha .
$$

Where $\beta\lfloor n$ denotes the restriction of $\beta$ in its first $n$ coordinates. For $\alpha \in$ $I^{n}$, say $\alpha=\left(i_{1}, \ldots, i_{n}\right)$, define $s_{\alpha}^{\lambda}: \mathbb{R}^{2} \rightarrow \mathbb{R}^{2}$ through iteration

$$
s_{\alpha}^{\lambda}=s_{i_{1}}^{\lambda} \circ s_{i_{2}}^{\lambda} \circ \cdots \circ s_{i_{n}}^{\lambda} .
$$

Let $A$ be the equilateral triangle with vertices $(0,0),(1,0),(1 / 2, \sqrt{3} / 2)$. Denote $s_{\alpha}^{\lambda}(A)=S_{\alpha}^{\lambda}, I^{0}=\{0\}$ and $s_{0}^{\lambda}=\mathrm{id}$. The limit set of the iteration

$$
E_{\lambda}=\bigcap_{j \geq 0} \bigcup_{\alpha \in I^{j}} S_{\alpha}^{\lambda}
$$


is self similar and in fact it is an $\lambda$-Sierpinski triangle with Hausdorff dimension

$$
d_{\lambda}=\operatorname{dim}_{\mathcal{H}} E_{\lambda}=-\frac{\log 3}{\log \lambda} .
$$

Notice that for $\lambda \in(0,1 / 3), d_{\lambda} \in(0,1)$. As a general property of self similar sets the measures $\mu_{\lambda}=\mathcal{H}^{d_{\lambda}}\left\lfloor E_{\lambda}\right.$ are $d_{\lambda}$ - AD regular. Hence there exists a constant $C_{\lambda}$, depending only on $\lambda$, such that for $x \in E_{\lambda}$ and $0<r \leq 1$

$$
C_{\lambda}^{-1} r^{d_{\lambda}} \leq \mu_{\lambda}(B(x, r)) \leq C_{\lambda} r^{d_{\lambda}} .
$$

The spaces $\left(E_{\lambda}, \rho, \mu_{\lambda}\right)$, where $\rho$ is the usual Euclidean metric, are simple examples of spaces of homogeneous type (see $[\mathbf{C h}]$ for definition). We want to find Calderón-Zygmund standard kernels on $E_{\lambda} \times E_{\lambda} \backslash\{(x, y)$ : $x=y\}$ that define bounded singular integral operators on $L^{2}\left(\mu_{\lambda}\right)$. In that direction, for $\lambda \in(0,1 / 3)$, we need to define two auxiliary families of functions.

The functions $\boldsymbol{\Omega}_{\boldsymbol{\lambda}}$. For any pair $(x, y) \in \mathbb{R}^{2} \times \mathbb{R}^{2}, x \neq y$, denote by $\theta_{(x, y)} \in[0,2 \pi)$ the angle formed by the vectors $y-x$ and $e_{1}=(1,0)$. For every $\lambda \in(0,1 / 3)$ there exists some positive number $\varepsilon_{\lambda}$ such that

(i) For all $x, y \in E_{\lambda}, x \neq y$

$$
\theta_{(x, y)} \in\left(\frac{k \pi}{3}-\varepsilon_{\lambda}, \frac{k \pi}{3}+\varepsilon_{\lambda}\right) \text { for some } k \in\{0,1, \ldots, 5\} .
$$

(ii) The intervals $\left(\frac{k \pi}{3}-\varepsilon_{\lambda}, \frac{k \pi}{3}+\varepsilon_{\lambda}\right)$ are disjoint for $k \in\{0,1, \ldots, 5\}$.

(iii) For any $n \in \mathbb{N}=\{1,2, \ldots\}$ and $\alpha, \beta, \gamma \in I^{n}, \alpha \neq \beta \neq \gamma$, such that $\alpha\lfloor n-1=\beta\lfloor n-1=\gamma\lfloor n-1$ :

(a) If $x \in S_{\alpha}^{\lambda}, y \in S_{\beta}^{\lambda}, z \in S_{\gamma}^{\lambda}$ and $\theta_{(x, y)} \in\left(\frac{k \pi}{3}-\varepsilon_{\lambda}, \frac{k \pi}{3}+\varepsilon_{\lambda}\right)$, for some $k \in\{0,1, \ldots, 5\}$, then $\theta_{(x, z)} \in\left(\frac{m \pi}{3}-\varepsilon_{\lambda}, \frac{m \pi}{3}+\varepsilon_{\lambda}\right)$ for $m=(k+1) \bmod 6$ or $m=(k-1) \bmod 6$.

(b) If $x, z \in S_{\alpha}^{\lambda}, y \in S_{\beta}^{\lambda}$ and $\theta_{(x, y)} \in\left(\frac{k \pi}{3}-\varepsilon_{\lambda}, \frac{k \pi}{3}+\varepsilon_{\lambda}\right)$ then $\theta_{(z, y)} \in\left(\frac{k \pi}{3}-\varepsilon_{\lambda}, \frac{k \pi}{3}+\varepsilon_{\lambda}\right)$ as well.

Now we can define $C^{\infty}$ functions $\Omega_{\lambda}$ on $S^{1}$ satisfying

(i) $\Omega_{\lambda}(z)=(-1)^{k}$ for $\theta_{(z, 0)} \in\left(\frac{k \pi}{3}-\varepsilon_{\lambda}, \frac{k \pi}{3}+\varepsilon_{\lambda}\right), k \in\{0,1, \ldots, 5\}$,

(ii) $\Omega_{\lambda}(-z)=-\Omega_{\lambda}(z)$ for every $z \in S^{1}$. 
Observe that the second condition also implies

$$
\int_{S^{1}} \Omega(z) d \sigma z=0
$$

where $\sigma$ is the normalized surface measure on $S^{1}$.

The functions $\boldsymbol{h}_{\boldsymbol{\lambda}}$. Fix some $\lambda \in(0,1 / 3)$, and choose any function $h_{\lambda}:(0, \infty) \rightarrow \mathbb{R}$ with the following properties,

(i) $h_{\lambda}$ is $C^{\infty}$,

(ii) $h_{\lambda}$ is increasing,

(iii) $h_{\lambda} L\left[\left(\frac{1}{\lambda}-2\right) \lambda^{k}, \lambda^{k-1}\right]=\lambda^{(k-1) d_{\lambda}}$ for every $k \in \mathbb{N}$.

It follows that for $r \in(0,1], h_{\lambda}(r) \approx r^{d_{\lambda}}$. In fact

$$
r^{d_{\lambda}} / C_{\lambda} \leq h_{\lambda}(r) \leq C_{\lambda} r^{d_{\lambda}} \text { for } 0<r \leq 1
$$

where $C_{\lambda}=\lambda^{-d_{\lambda}}$.

Hence we are able, using the above families, to define appropriate kernels

$$
K_{\lambda}: E_{\lambda} \times E_{\lambda} \backslash\{(x, y): x=y\} \rightarrow \mathbb{R}
$$

as

$$
K_{\lambda}(x, y)=\frac{\Omega_{\lambda}((x-y) /|x-y|)}{h_{\lambda}(|x-y|)} .
$$

For the kernels $K_{\lambda}$ there exists some constant $C$ such that for all $x, y, z \in$ $E_{\lambda}, x \neq y$, satisfying $|x-z|<(1-2 \lambda)|x-y|$,

$$
\begin{aligned}
& \left|K_{\lambda}(x, y)\right| \leq \frac{C}{|x-y|^{d_{\lambda}}}, \\
& K_{\lambda}(x, y)-K_{\lambda}(z, y)=0 .
\end{aligned}
$$

Condition (2.1) follows immediately from the definition of $K_{\lambda}$. To prove (2.2), let $k \in \mathbb{N}^{*}=\{0,1, \ldots\}$ be the largest natural number such that $x, y \in S_{\alpha}^{\lambda}$ for some $\alpha \in I^{k}$. Therefore

$$
x \in s_{i}^{\lambda}\left(S_{\alpha}^{\lambda}\right) \text { and } y \in s_{j}^{\lambda}\left(S_{\alpha}^{\lambda}\right)
$$

for some $i, j \in I, i \neq j$. This implies that,

$$
\left(\frac{1}{\lambda}-2\right) \lambda^{k+1} \leq|y-x| \leq \lambda^{k}
$$

Since $|x-z|<(1-2 \lambda)|x-y|$ we get

$$
|x-z|<(1-2 \lambda) \lambda^{k}
$$


As

$$
d\left(s_{i}^{\lambda}\left(S_{\alpha}^{\lambda}\right), s_{q}^{\lambda}\left(S_{\alpha}^{\lambda}\right)\right)=(1-2 \lambda) \lambda^{k} \text { for } q \in I, q \neq i,
$$

and

$$
S_{\alpha}^{\lambda}=\bigcup_{p \in I} s_{p}^{\lambda}\left(S_{\alpha}^{\lambda}\right)
$$

we deduce that $z \in s_{i}^{\lambda}\left(S_{\alpha}^{\lambda}\right)$ and

$$
\left(\frac{1}{\lambda}-2\right) \lambda^{k+1} \leq|y-z| \leq \lambda^{k} .
$$

Therefore as $x, z \in s_{i}^{\lambda}\left(S_{\alpha}^{\lambda}\right)$ and $y \in s_{j}^{\lambda}\left(S_{\alpha}^{\lambda}\right)$

$$
\theta(x, y), \theta(z, y) \in\left(m \frac{\pi}{3}-\varepsilon_{\lambda}, m \frac{\pi}{3}+\varepsilon_{\lambda}\right)
$$

for some $m \in\{0,1, \ldots, 5\}$. From (2.3), (2.4), (2.5) and the definition of $h_{\lambda}$ and $\Omega_{\lambda}$ we deduce that

$$
h_{\lambda}(|x-y|)=h_{\lambda}(|z-y|)=\lambda^{k d_{\lambda}},
$$

and

$$
\Omega_{\lambda}\left(\frac{x-y}{|x-y|}\right)=\Omega_{\lambda}\left(\frac{z-y}{|z-y|}\right) .
$$

Hence

$$
K_{\lambda}(x, y)-K_{\lambda}(z, y)=0
$$

and by antisymmetry

$$
K_{\lambda}(y, x)-K_{\lambda}(y, z)=0 .
$$

It follows that the kernels $K_{\lambda}$ are CZ standard, in fact condition (2.2) is much stronger than the ones appearing in the usual definitions of $\mathrm{CZ}$ standard kernels (see e.g. $[\mathbf{C h}],[\mathbf{D} \mathbf{1}]$ and $[\mathbf{J}]$ ).

As stated before, we want to show that the kernels define singular integral operators that are bounded in $L^{2}\left(\mu_{\lambda}\right)$, and examine their convergence properties.

\section{3. $L^{2}$ boundedness}

Theorem 3.1. For all $\lambda \in(0,1 / 3)$ the maximal singular integral operators $T_{\lambda}^{*}$,

$$
T_{\lambda}^{*}(f)(x)=\sup _{\varepsilon>0}\left|\int_{|x-y|>\varepsilon} K_{\lambda}(x, y) f(y) d \mu_{\lambda} y\right|,
$$

are bounded in $L^{2}\left(\mu_{\lambda}\right)$. 
Proof: The idea is to use the T(1) theorem of David and Journé in the context of [D2]. Start by defining $T_{\lambda}^{n}$, for $n \geq 1$, as

$$
T_{\lambda}^{n}(f)(x)=\int_{|x-y|>\lambda^{n}} K_{\lambda}(x, y) f(y) d \mu_{\lambda} y
$$

We want to show that $T_{\lambda}^{n}(\mathbf{1})=0$ for all $n \in \mathbb{N}$, by induction.

For $n=1$ : Let $x \in S_{i}^{\lambda} \cap E_{\lambda}$ for some $i \in I$. If $j, k \in I \backslash\{i\}, j \neq k$, we get

$$
\begin{aligned}
T_{\lambda}^{1}(\mathbf{1})(x) & =\int_{|x-y|>\lambda} K_{\lambda}(x, y) d \mu_{\lambda} y \\
& =\int_{S_{j}^{\lambda} \cup S_{k}^{\lambda}} K_{\lambda}(x, y) d \mu_{\lambda} y \\
& =\int_{S_{j}^{\lambda}} \frac{\Omega_{\lambda}((x-y) /|x-y|)}{h_{\lambda}(|x-y|)} d \mu_{\lambda} y+\int_{S_{k}^{\lambda}} \frac{\Omega_{\lambda}((x-y) /|x-y|)}{h_{\lambda}(|x-y|)} d \mu_{\lambda} y .
\end{aligned}
$$

Furthermore there exists some $m \in\{0,1, \ldots, 5\}$ such that for $y \in S_{j}^{\lambda}$,

$$
\Omega_{\lambda}\left(\frac{x-y}{|x-y|}\right)=(-1)^{m}
$$

and for $y \in S_{k}^{\lambda}$,

$$
\Omega_{\lambda}\left(\frac{x-y}{|x-y|}\right)=(-1)^{m+1}
$$

Hence

$T_{\lambda}^{1}(\mathbf{1})(x)=(-1)^{m} \int_{S_{j}^{\lambda}} \frac{1}{h_{\lambda}(|x-y|)} d \mu_{\lambda} y+(-1)^{m+1} \int_{S_{k}^{\lambda}} \frac{1}{h_{\lambda}(|x-y|)} d \mu_{\lambda} y$.

But for $y \in S_{j}^{\lambda} \cup S_{k}^{\lambda}$ we have that $1-2 \lambda \leq|x-y| \leq 1$ and consequently $h_{\lambda}(|x-y|)=1$. Thus

$$
T_{\lambda}^{1}(\mathbf{1})(x)=(-1)^{m} \mu_{\lambda}\left(S_{j}^{\lambda}\right)+(-1)^{m+1} \mu_{\lambda}\left(S_{k}^{\lambda}\right)=0 .
$$

Suppose that $T_{\lambda}^{n}(\mathbf{1})=0$ and let some $x \in E_{\lambda}$. We want to show that $T_{\lambda}^{n+1}(\mathbf{1})(x)=0$. Let $x \in S_{\alpha}^{\lambda}$ for some $\alpha=\left(i_{1}, i_{2}, \ldots, i_{n}, i_{n+1}\right) \in I^{n+1}$. 
If $\beta=\left(i_{1}, i_{2}, \ldots, i_{n}, j\right)$ and $\gamma=\left(i_{1}, i_{2}, . . i_{n}, k\right)$ for $j, k \in I \backslash\left\{i_{n+1}\right\}, j \neq k$,

$$
\begin{aligned}
T_{\lambda}^{n+1}(\mathbf{1})(x)= & \int_{|x-y|>\lambda^{n+1}} K_{\lambda}(x, y) d \mu_{\lambda} y \\
= & \int_{|x-y|>\lambda^{n}} K_{\lambda}(x, y) d \mu_{\lambda} y+\int_{S_{\beta}^{\lambda}} K_{\lambda}(x, y) d \mu_{\lambda} y \\
& +\int_{S_{\gamma}^{\lambda}} K_{\lambda}(x, y) d \mu_{\lambda} y \\
= & \int_{S_{\beta}^{\lambda}} \frac{\Omega_{\lambda}((x-y) /|x-y|)}{h_{\lambda}(|x-y|)} d \mu_{\lambda} y \\
& +\int_{S_{\gamma}^{\lambda}} \frac{\Omega_{\lambda}((x-y) /|x-y|)}{h_{\lambda}(|x-y|)} d \mu_{\lambda} y
\end{aligned}
$$

since by the induction hypothesis

$$
T_{\lambda}^{n}(\mathbf{1})(x)=\int_{|x-y|>\lambda^{n}} K_{\lambda}(x, y) d \mu_{\lambda} y=0 .
$$

Using exactly the same argument as in the case for $n=1$

$$
\int_{S_{\beta}^{\lambda}} \frac{\Omega_{\lambda}\left((x-y) /|x-y|^{-1}\right)}{h_{\lambda}(|x-y|)} d \mu_{\lambda} y+\int_{S_{\gamma}^{\lambda}} \frac{\Omega_{\lambda}\left((x-y) /|x-y|^{-1}\right)}{h_{\lambda}(|x-y|)} d \mu_{\lambda} y=0 .
$$

Therefore $T_{\lambda}^{n+1}(\mathbf{1})(x)=0$, completing the induction. As $T_{\lambda}^{n}(\mathbf{1})=0$ for all $n \in \mathbb{N}$ the same holds for their transposes.

Due to the structure of the spaces $\left(E_{\lambda}, \mu_{\lambda}, \rho\right)$ the proof of the $T(1)$ theorem in this setting is essentially the same with the one appearing in $[\mathbf{D 1}$. As commented in [D1] and in [D2], in order to be able to use the $T(1)$ theorem we need some suitable decomposition of dyadic type, as in $[\mathbf{C h}]$ and $[\mathbf{D 1}]$, to replace the usual dyadic cubes in $\mathbb{R}^{n}$. In our setting the required such family $\mathcal{R}$ consists of all the triangles appearing in every step of the iteration process, i.e.

$$
\mathcal{R}_{k}^{\lambda}=\left\{S_{\alpha}^{\lambda}: \alpha \in I^{k}\right\} \text { for } k \in \mathbb{N}^{*},
$$

and

$$
\mathcal{R}=\left\{\mathcal{R}_{k}^{\lambda}: k \in \mathbb{N}^{*}\right\}
$$


In the assumptions of the original David-Journé $T(1)$ theorem the operators should also satisfy an extra condition the so called weak boundedness. This condition is only used in the proof, as it appears in [D1], to show that there exists some absolute constant $C$, such that for all dyadic cubes $Q$

$$
\left|\int_{Q} T\left(\mathbf{1}_{Q}\right)(x) d x\right| \leq C|Q| .
$$

But since the operators $T_{\lambda}^{n}$ are canonically associated with antisymmetric kernels the weak boundedness comes for free, see e.g. $[\mathbf{C h}]$.

Applying the $T(1)$ theorem we derive that every element of the sequence $\left\{T_{\lambda}^{n}\right\}_{n \in \mathbb{N}}$ is bounded in $L^{2}\left(\mu_{\lambda}\right)$ with bounds not depending on $n$. This fact enables us to extract some linear $L^{2}\left(\mu_{\lambda}\right)$-bounded operator $T$ as a weak limit some subsequence of $\left\{T_{\lambda}^{n}\right\}_{n \in \mathbb{N}}$. Finally using the version of Cotlar's inequality, as it is stated in [D1, p. 59], we get that there exists some constant $C$ such that for all $f \in L^{2}\left(\mu_{\lambda}\right)$

$$
T_{\lambda}^{*}(f)(x) \leq C\left(M_{\lambda}(T f)(x)+M_{\lambda}\left(|f|^{\sqrt{2}}(x)\right)^{\sqrt{2}}\right),
$$

where $M_{\lambda}$ is the Hardy-Littlewood maximal operator related to the measure $\mu_{\lambda}$. Therefore we conclude that $T_{\lambda}^{*}$ is bounded in $L^{2}\left(\mu_{\lambda}\right)$.

\section{Divergence of principal values}

Theorem 4.1. Let $\lambda \in(0,1 / 3)$. For $\mu_{\lambda}$ almost every point in $E_{\lambda}$ the principal values of the singular integral operator $T_{\lambda}$ do not exist.

Proof: Let $\lambda \in(0,1 / 3)$, we want to show that for $\mu_{\lambda}$ a.e. $x \in E_{\lambda}$ the limit

$$
\lim _{\varepsilon \rightarrow 0}\left|\int_{\mathbb{R}^{2} \backslash B(x, \varepsilon)} K_{\lambda}(x, y) d \mu_{\lambda} y\right|
$$

does not exist.

To every $z \in E_{\lambda}$ assign naturally the code $\left(z_{i}\right)_{i \in \mathbb{N}} \in I^{\infty}$ such that $\{z\}=\bigcap_{i \geq 1} S_{\left(z_{1}, \ldots, z_{i}\right)}^{\lambda}$ and consider the set

$$
D_{\lambda}=\left\{z \in E_{\lambda}: z_{i} \neq z_{i+1} \text { for infinitely many } i \text { 's }\right\} .
$$

The set $D_{\lambda}$ had full $\mu_{\lambda}$ measure as its complement $E_{\lambda} \backslash D_{\lambda}$ is countable. In fact the set $E_{\lambda} \backslash D_{\lambda}$ consists of the vertices of every triangle $S_{\alpha}^{\lambda}, \alpha \in I^{*}$. 


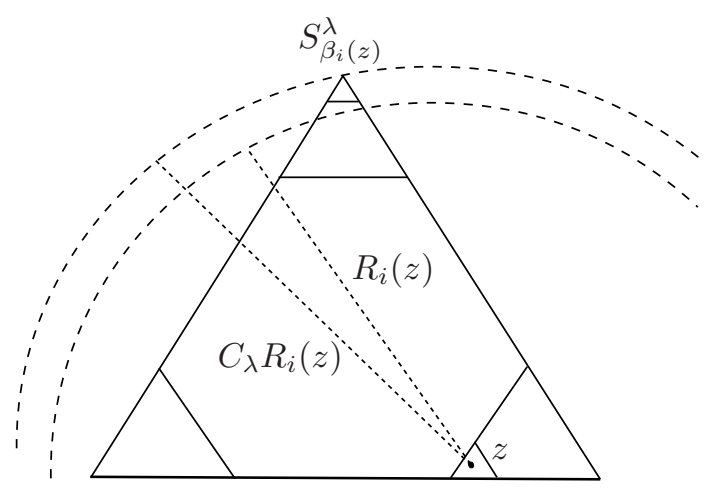

Figure A

Notice that there exist some $C_{\lambda}>1$ and some $m_{\lambda} \in \mathbb{N}$ such that for every $z \in D_{\lambda}$ and every $i \in \mathbb{N}^{*}$, satisfying $z_{i} \neq z_{i+1}$, there exist $\beta_{i}(z) \in I^{i-1+m_{\lambda}}$ and positive numbers $R_{i}(z)$ with the properties,

(i) $\beta_{i}(z)=(z_{1}, \ldots, z_{i-1}, \overbrace{y(z), \ldots, y(z)}^{m_{\lambda} \text { times }})$ where $y(z) \in I \backslash\left\{z_{i}, z_{i+1}\right\}$,

(ii) $R_{i}(z) \approx \lambda^{i}$,

(iii) $B\left(z, C_{\lambda} R_{i}(z)\right) \backslash B\left(z, R_{i}(z)\right) \cap E_{\lambda}=S_{\beta_{i}(z)}^{\lambda}$.

See also Figure A. This geometric property of the sets $E_{\lambda}$ forces the principal values of $T_{\lambda}$ to diverge.

To see this, let some $x \in D_{\lambda}$ and denote $J_{x}=\left\{i \in \mathbb{N}^{*}: x_{i} \neq x_{i+1}\right\}$. For all $i \in J_{x}$,

$$
\begin{array}{r}
\left|\int_{\mathbb{R}^{2} \backslash B\left(x, R_{i}(x)\right)} K_{\lambda}(x, y) d \mu_{\lambda} y-\int_{\mathbb{R}^{2} \backslash B\left(x, C_{\lambda} R_{i}(x)\right)} K_{\lambda}(x, y) d \mu_{\lambda} y\right| \\
=\left|\int_{B\left(x, C_{\lambda} R_{i}(x)\right) \backslash B\left(x, R_{i}(x)\right)} \frac{\Omega_{\lambda}\left((x-y)|x-y|^{-1}\right)}{h_{\lambda}(|x-y|)} d \mu_{\lambda} y\right| \\
=\left|\int_{S_{\beta_{i}(x)}^{\lambda}} \frac{\Omega_{\lambda}\left((x-y)|x-y|^{-1}\right)}{h_{\lambda}(|x-y|)} d \mu_{\lambda} y\right| .
\end{array}
$$

For all $x \in S_{a_{i}(x)}^{\lambda}$ and $y \in S_{\beta_{i}(x)}^{\lambda}$, where $\alpha_{i}(x)=\left(x_{1}, \ldots, x_{i}, x_{i+1}\right)$,

$$
(1-2 \lambda) \lambda^{i-1} \leq|x-y| \leq \lambda^{i-1}
$$


and

$$
\Omega_{\lambda}\left(\frac{x-y}{|x-y|}\right)=(-1)^{\varepsilon_{i}}
$$

where $\varepsilon_{i}=1$ or $\varepsilon_{i}=-1$. Hence

$$
\begin{aligned}
\left|\int_{S_{\beta_{i}(x)}} \frac{\Omega_{\lambda}\left((x-y)|x-y|^{-1}\right)}{h_{\lambda}(|x-y|)} d \mu_{\lambda} y\right| & =\int_{S_{\beta_{i}(x)}^{\lambda}} \frac{1}{h_{\lambda}(|x-y|)} d \mu_{\lambda} y \\
& =\frac{\mu_{\lambda}\left(S_{\beta_{i}(x)}^{\lambda}\right)}{\left(\lambda^{i-1}\right)^{d_{\lambda}}} \\
& =\frac{\left(\lambda^{i-1+m_{\lambda}}\right)^{d_{\lambda}}}{\left(\lambda^{i-1}\right)^{d_{\lambda}}}=\lambda^{m_{\lambda} d_{\lambda}} .
\end{aligned}
$$

As $R_{i}(x) \approx \lambda^{i} \rightarrow 0$ we conclude that the principal values of $T_{\lambda}$ do not exist $\mu_{\lambda}$ a.e.

Acknowledgements. I am very grateful to my advisor, Professor Pertti Mattila, for many ideas, discussions and suggestions during the preparation of this note. I would also like to thank the referee for useful comments and suggestions.

\section{References}

[Ch] M. CHRIST, "Lectures on singular integral operators", CBMS Regional Conference Series in Mathematics 77, Published for the Conference Board of the Mathematical Sciences, Washington, DC; by the American Mathematical Society, Providence, RI, 1990.

[D1] G. DAVID, "Wavelets and singular integrals on curves and surfaces", Lecture Notes in Mathematics 1465, Springer-Verlag, Berlin, 1991.

[D2] G. DAVID, Des intégrales singulières bornées sur un ensemble de Cantor, C. R. Acad. Sci. Paris Sér. I Math. 332(5) (2001), 391-396.

[DJ] G. DAVID AND J.-L. JouRnÉ, A boundedness criterion for generalized Calderón-Zygmund operators, Ann. of Math. (2) 120(2) (1984), 371-397.

[Hu] P. Huovinen, A nicely behaved singular integral on a purely unrectifiable set, Proc. Amer. Math. Soc. 129(11) (2001), 3345-3351 (electronic). 
[J] J.-L. JouRné, "Calderón-Zygmund operators, pseudodifferential operators and the Cauchy integral of Calderón", Lecture Notes in Mathematics 994, Springer-Verlag, Berlin, 1983.

[MM] P. Mattila and M. S. Melnikov, Existence and weak-type inequalities for Cauchy integrals of general measures on rectifiable curves and sets, Proc. Amer. Math. Soc. 120(1) (1994), $143-149$

[MMV] P. Mattila, M. S. Melnikov, and J. Verdera, The Cauchy integral, analytic capacity, and uniform rectifiability, Ann. of Math. (2) 144(1) (1996), 127-136.

[MV] P. Mattila and J. Verdera, Convergence of singular integrals with general measures, J. Eur. Math. Soc. (JEMS) (to appear).

[Pr] L. PRAT, Principal values for the signed Riesz kernels of noninteger dimension, submitted.

[T] X. TolsA, Cotlar's inequality without the doubling condition and existence of principal values for the Cauchy integral of measures, J. Reine Angew. Math. 502 (1998), 199-235.

Department of Mathematics and Statistics

P. O. Box 68

FI-00014 University of Helsinki

Finland

E-mail address: vasileios.chousionis@helsinki.fi

Primera versió rebuda el 6 de febrer de 2008, darrera versió rebuda el 14 d'abril de 2008. 